\section{4-S1.02 MOLECULAR CLONING AND EXPRESSION OF HYDROGENOSOMAL MALATE DEHYDROGENASE OF TRICHOMONAS VAGINALIS}

doi:10.1136/sextrans-2011-050109.140

${ }^{1} \mathrm{~S}$ Ardalan, ${ }^{2} \mathrm{C}$ Lee, ${ }^{2} \mathrm{G}$ Garber. ${ }^{1}$ Faculty of Medicine, University of Ottawa, The Ottawa Hospital Research Institute Ottawa, Canada; ${ }^{2}$ Faculty of Medicine, University of Ottawa, Division of Infectious Diseases, The Ottawa Hospital Ottawa, Canada

Background Trichomoniasis, a sexually transmitted disease caused byTrichomonas vaginalis, is associated with adverse pregnancy outcomes, and increased risk of HIV acquisition. Malate dehydrogenase $(\mathrm{MDH})$, which catalyses the interconversion of malate to oxaloacetate, has a pivotal role in the survival and pathogenicity of this amitochondrial protozoan. The objective of this study was to clone and express Malate dehydrogenase gene of $T$ vaginalis, and analyse the biological function of this hydrogenosomal enzyme.

Methods The $\mathrm{MDH}$ gene from a clinical isolate of $T$ vaginalis was amplified by PCR, and cloned into pET101/D-TOPO vector with a C-terminal 6XHis tag. Positive clones were screened and identified by restriction endonuclease digestion and sequence analysis. The plasmid pET101/D-MDH was then transformed into E.coli BL21 (DE3) to express after IPTG induction. The expression product further analysed by sodium dodecyl sulphate polyacrylamide gel electrophoresis (SDS-PAGE) and Western blotting. The recombinant protein was purified with Ni-NTA agarose under native conditions. Western blot, using antibody raised against whole cellT vanginalis, was performed to determine the immunogenicity of purified recombinant protein.

Results The recombinant plasmid pET101/D-MDH was constructed successfully. High homology (98\%) of nucleotide sequence was revealed between the cloned $\mathrm{MDH}$ and the corresponding gene. The recombinant protein showed a high expression level when induced with $1 \mathrm{mM}$ IPTG at $37^{\circ} \mathrm{C}$ for $4 \mathrm{~h}$. SDS-PAGE analysis showed that the recombinant $\mathrm{MDH}$ protein with the correct molecular weight (about $60 \mathrm{kDa}$ ) was expressed in E.coli BL21 (DE3). Western blotting revealed that the purified recombinant protein was specifically recognised by sera from mice infected with whole cell $T$ vaginalis.

Conclusions A prokaryotic expression system of T vaginalis Malate dehydrogenase gene has been established successfully. The immunogenicity of the recombinant protein has been tested. The present study shows that the recombinant $\mathrm{MDH}$ is specific and suitable for use as an antigen for detecting anti-Trichomonas vaginalis IgG antibodies. Our work has established a good foundation for future studies on $T$ vaginalis vaccine construction.

\section{4-S1.03 DEFINING THE IN VITRO FUNCTIONS OF MONOCLONAL ANTIBODIES DEVELOPED TO THE HAEMOPHILUS DUCREYI TRIMERIC AUTOTRANSPORTER DSRA}

doi:10.1136/sextrans-2011-050109.141

I Leduc. UNC at Chapel Hill, Chapel Hill, USA

Background The DsrA protein ofHaemophilus ducreyi, the etiological agent of the genital ulcer disease chancroid, is a multifunctional outer membrane trimeric autotransporter (TA) and a virulence factor. Most $H$ ducreyi strains, which are grouped in two classes (I and II), express a DsrA protein. Although DsrA proteins from both classes of $H$ ducreyi strains share little identity in their N-terminal functional passenger domain, both are involved in serum resistance and function as adhesins to the extracellular matrix (ECM) proteins fibronectin (Fn) and vitronectin ( $\mathrm{Vn}$ ), as well as to fibrinogen (Fbg) and $\mathrm{HaCat}$ keratinocytes.

Methods Monoclonal antibodies (mAbs) to DsrA were developed by immunising mice with full-length recombinant DsrA from class I strain $35000 \mathrm{HP}$ (DsrAI). Western blots were used to determine the specificity of the mAbs and their ability to recognise multimers of DsrA. Whole-cell binding ELISAs were used to examine the capacity of the mAbs to bind the surface of $H$ ducreyi. The domains and the shortest nominal peptides of DsrA recognised by those mAbs were identified using a library of truncated DsrA proteins expressed in a dsrA mutant, a peptide library representing full-length DsrAI, as well as Surface Plasmon Resonance and mutagenesis studies. mAbs were tested for bactericidal activity and their ability to block binding of Fn, $\mathrm{Vn}$ and $\mathrm{Fbg}$ byH ducreyi using bactericidal assays and whole cell blocking assays, respectively.

Results Anti-DsrAI mAbs bound monomers and dimers of the DsrA protein from several class $\mathrm{I} H$ ducreyi strains but not the DsrAII protein. $\mathrm{mAb} 1.82$ bound native DsrAI protein at the surface of a panel of class $\mathrm{I} H$ ducreyi isolates, but not the $\mathrm{H}$.ducreyi strains that cause cutaneous chancroid. The DsrA protein from these strains was recognised by $\mathrm{mAb} 4.79$, along with the DsrA protein from strain $35000 \mathrm{HP}$. Both 1.82 and 4.79 bound with high affinity to peptides in the N-terminal region of the DsrA translocator domain and the shortest nominal epitope for 1.82 is MEQNTHNINKLS. In whole cell blocking assays, mAb 1.82 partially blocked binding of Fn and Fbg by class $\mathrm{I} H$ ducreyi strain $35000 \mathrm{HP}$.

Conclusions 1.82 and 4.79 are DsrAI-specific mAbs that recognise multimers of DsrA and bind the surface of intact $H$ ducreyi. Both $\mathrm{mAbs}$ bind an epitope in the $\mathrm{N}$-terminal region of the translocator domain of DsrAI with high affinity. mAb 1.82 has modest Fn and Fbg blocking activity.

\section{4-S1.04 RAPID SPREAD OF HERPES SIMPLEX VIRUS-2 IN THE HUMAN GENITAL TRACT}

doi:10.1136/sextrans-2011-050109.142

${ }^{1} \mathrm{~J}$ Schiffer, ${ }^{2} \mathrm{D}$ Swan, ${ }^{3} \mathrm{~A}$ Magaret, ${ }^{3} \mathrm{C}$ Johnston, ${ }^{3} \mathrm{~S}$ Selke, ${ }^{3} \mathrm{~A}$ Wald, ${ }^{1} \mathrm{~L}$ Corey. ${ }^{1}$ Fred Hutchinson Cancer Research Center Seattle, Washington, USA; ${ }^{2}$ Fed Hutchinson Cancer Research Center, USA; ${ }^{3}$ University of Washington, USA

Background Genital herpes simplex virus-2 (HSV-2) infection consists of shedding episodes that correlate with transmission and genital lesion formation. Episodes are heterogeneous in terms of viral production and duration.

Methods Using quantitative PCR data from 14685 daily genital swabs performed daily in 531 persons, we summarised 1020 episodes according to their frequency, duration, peak HSV DNA copy number, first and last swab copy number, expansion and decay slopes, and morphology. We designed competing dynamical stochastic mathematical models and attempted to fully reproduce result ranges for each of these episode features. The models assumed the possibility of concurrent, spatially distinct plaques of viral infection and immunologic response.

Results Our spatial model reproduced all kinetic features of the empirical shedding data. The model and parameter set that achieved best fit highlights three distinct mechanisms of viral spread. First, HSV-2 is constantly dispersed from neurons to epidermal cells throughout the genital tract at a rate of $\sim 100$ HSV DNA copies/ day. Approximately 75 times each year, a neuron-derived viral particle infects an epidermal cell, initiating an infectious plaque of epidermal cells and a detectable shedding episode. Second, within each plaque, cell-to-cell spread of HSV-2 particles occurs extremely rapidly between epidermal cells. One cell can produce 105 cellassociated HSV genomic copies/day, with $3 \%$ of viral particles infecting a neighbouring cell each day. In large plaques, $>105$ cells may be infected within $\sim 12 \mathrm{~h}$ producing up to 109 HSV DNA copies/ml. Third, cell-free viruses, despite being 3000-times less infectious than cell-associated viruses, decay more slowly and initiate spatially distinct secondary plaques. We use video simulations to display that episodes longer than 3 days, consist of dozens 
of concurrent viral plaques. Despite rapid cell-to-cell spread of HSV2 , infected cells are eliminated by localised CD8 + T-cells within $24 \mathrm{~h}$ of plaque initiation. Moreover, the extent of secondary plaque formation prior to episode termination is determined by spatial CD8 + T-cell density surrounding the site of infection.

Conclusions Genital HSV-2 utilises three kinetically distinct methods of spread to initiate and sustain prolonged shedding episodes. The extent and severity of secondary plaque formation is determined by spatial immune cell density.

\section{4-S1.05 RNA HELICASES, P72 AND P68 AND HDAC1 INTERACT WITH HIV-1 INTEGRASE AND AFFECT VIRAL REPLICATION}

doi:10.1136/sextrans-2011-050109.143

F Larguet, É Rassart, E Edouard. Université du Québec à Montréal, Montreal, Canada

Background Human immunodeficiency virus type-1 (HIV-1) infection is one of the leading causes of death worldwide. Current antiHIV-1 therapy, referred as highly active antiretroviral therapy (HAART), is based on the use of combination of drugs directed against viral enzymes mainly reverse transcriptase and protease and more recently integrase. Indeed, HAART has dramatically improved the clinical course of the disease. However, the emergence of multidrug resistant virus strains during treatment highlights the urgent need to develop novel antiretroviral drugs against new HIV-1 targets. HIV-1 is able to hijack cellular machinery for its replication through protein-protein interactions between viral and host cell factors and a rising strategy against HIV-1 infection is to inhibit key virus-cell interactions. Integrase that catalyses HIV-1 viral DNA integration into the host cell genome is currently a focus for the development of new drugs. Several cellular partners of integrase have been identified using different methods. Based on a different strategy, our study aimed to identify new integrase cellular partners. Methods We used a biotinylated oligonucleotide derived from the viral U3 LTR end as bait to isolate integrase in a streptavidin beads magnetic separation. Proteins co-purified with integrase were analysed by mass spectrometry.

Results Interestingly, our method allowed the identification of new cellular proteins notably p72 and p68 RNA helicases and histone deacetylase 1 (HDAC1) as integrase partners in addition to proteins already reported in literature. The interaction of p72, p68 and HDAC1 proteins with integrase was confirmed by co-immunoprecipitation. In addition, specific knockdown of p72 and p68 RNA helicases and HDAC1 were shown to affect HIV-1 replication.

Conclusions Our data suggest that cellular proteins, p72 and p68 RNA helicases and HDAC1 facilitate HIV-1 replication through interaction with integrase.

\section{4-S1.06 HPV 16 PREDICTS CLINICAL OUTCOME IN ORAL CANCER PATIENTS TREATED BY RADIOTHERAPY}

doi:10.1136/sextrans-2011-050109.144

R M Apolinario, P C Lara, L A Henriquez, M Lloret, B Pinar. Las Palmas University Hospital-Canarian Institute for Cancer Research (ICIC), Las Palmas GC, Spain

Growing molecular and clinical evidence indicates that human papillomavirus (HPV) is involved in the aetiology of oral squamous cell carcinomas (SCCs). HPV (+) tumours appear to be clinically distinct from $\mathrm{HPV}(-)$ tumours, conferring improved survival outcomes for patients in oropharyngeal cancer but limited knowledge exist on oral cancer. Determination of the HPV status of tumours may assist in patient risk-stratification and ultimately guide optimum treatment. The primary aim of this study was to examine the distribution of HPV in oral SCCs as assessed in vitro amplification assays and correlated with clinical and demographic data. The secondary aim was to correlate the positivity of HPV tumours with clinical outcome in the largest series of oral cancer published with a long follow-up (up to 20 years).

Materials and Methods One hundred thirty-one invasive oral SCCs were tested for HPV using laboratory-developed PCR assays for HPV16. P53 expression, tumour angiogenesis (CD-31 staining) and proliferation (MIB-1) were also assessed by immunohistochemistry in parafine embedded tissue. Patients mean age was 58.09 \pm 10.41 , median 59 (116 men and 15 women). Clinical Stage distribution was: st I:17 cases; II: 56,III:32.IV:26. Most tumours were histological grade I (39) and II (74). Patients with pathological stage I-II were refereed to surgery (65 cases) and patients with Stage III-IVA were referred to surgery and postoperative radiation therapy (66 cases). Mean radiotherapy given doses were $62.13 \pm 7.74$, median $65 \mathrm{~Gy}$ in 1.8-2 Gy fractions. No chemotherapy was used in any case.

Results 41 cases $(31.3 \%)$ were HPV $16(+)$. No relation was found with age, gender, or tumour characteristics. In fact no relation was found to p53 expression, tumour proliferation or angiogenesis. 15year DFS was $62.20 \%$ in $\mathrm{HPV}(+)$ patients was, compared to $37.3 \%$ in the HPV(-) group $(p<0.076)$. In stage III-IV cases (treated by surgery and radiation therapy) this differences reached statistical signification ( 15 y DFS $72.4 \%$ vs $36.0 \%$ p <0.020). Similar results were found for Cause Especific Survival (15 y DFS 68.4\% vs $26.2 \%$ $\mathrm{p}<0.054)$.

Conclusion These data show that the HPV status is a good predictor of DFS and survival in patients treated with radical surgery and adjuvant radiotherapy in oral carcinomas. This prognostic advantage seem to be independent of tumour proliferation, p53 status or angiogenesis. Other molecular processes could be implicated in the different response to radiotherapy.

\section{Basic sciences oral session 2-Immunity and animal models \\ 04-S2.01 THE HOST RESPONSE TO CHLAMYDIAL INFECTION RESULTS IN INCREASED GONOCOCCAL COLONISATION IN A NOVEL COINFECTION MODEL}

doi:10.1136/sextrans-2011-050109.145

${ }^{1} \mathrm{~A}$ Jerse, ${ }^{1} \mathrm{R}$ Vonck, ${ }^{2} \mathrm{~T}$ Darville. ${ }^{1}$ Uniformed Services University, Bethesda, USA ${ }^{2}$ University of Pittsburgh Medical Center, Pittsburgh, USA

Objective Neisseria gonorrhoeae and Chlamydia trachomatis cause similar urogenital diseases and up to $70 \%$ of individuals with gonorrhoea also have chlamydia. Using a newly developed female mouse model of coinfection, we recently reported that higher numbers of $N$ gonorrhoeae were recovered from mice with a preexisting Chlamydia muridarum infection, the mouse strain of Chlamydia, compared to mice infected with $N$ gonorrhoeae alone. Recent studies on the host response to $N$ gonorrhoeae implicate toll-like receptor 4 (TLR4) and IL17 responses as being protective against $N$ gonorrhoeae. Here we tested the hypothesis that the immune response to chlamydial infection makes the genital tract more permissive to $N$ gonorrhoeae.

Methods Using an immune-targeted RT-PCR array we screened for alterations in host gene expression during chlamydial infection of $\mathrm{BALB} / \mathrm{c}$ mice that may account for the observed increase in gonococcal colonisation. Mouse genital cells were collected by vaginal swab and analysed for TLR4 expression by flow cytometry. Coinfection studies were performed in BALB/cJ (TLR4 wild type) and C. C3-TLR4LPS-d/J (TLR4 mutant) mice and the number of viable chlamydiae and gonococci recovered from each group was determined by immunofluorescence using $L 929$ cells and quantitative culture on GC agar, respectively. 